\title{
Conformational Preferences and Internal Rotation in Alkyl- and Phenyl-Substituted Thiourea Derivatives
}

\author{
Vyacheslav S. Bryantsev and Benjamin P. Hay* \\ Chemical Sciences Division. Pacific Northwest National Laboratory, P.O. Box 999, \\ Richland, Washington 99352.
}

Supporting Information Available. Cartesian coordinates and energies (Hartrees) for the MP2/aug-cc-pVDZ optimized equilibrium and transition state geometries, and a Table comparing calculated geometric data for $\mathbf{6}$ to that observed in X-ray crystal structures.

$\begin{array}{lccc}\mathbf{1} \text { anti conformer MP2/aug-cc-pVDZ } & \text { T.E. }=-547.320362486 \text { a.u. } \\ \mathrm{S} & 0.00000 & 0.00000 & 1.48838 \\ \mathrm{~N} & 0.01245 & -1.15089 & -0.92883 \\ \mathrm{C} & 0.00000 & 0.00000 & -0.17690 \\ \mathrm{H} & -0.37630 & -1.12189 & -1.86520 \\ \mathrm{H} & -0.14815 & -2.00331 & -0.40706 \\ \mathrm{~N} & -0.01245 & 1.15089 & -0.92883 \\ \mathrm{H} & 0.37630 & 1.12189 & -1.86520 \\ \mathrm{H} & 0.14815 & 2.00331 & -0.40706\end{array}$

1 syn conformer MP2/aug-cc-pVDZ T.E.= -547.319557638 a.u. (-232.4 cm-1)

$\begin{array}{llll}\mathrm{S} & 0.00286 & -1.35876 & 0.00000 \\ \mathrm{~N} & 0.01933 & 1.04791 & -1.15082 \\ \mathrm{C} & 0.00033 & 0.31253 & 0.00000 \\ \mathrm{H} & -0.09614 & 2.05246 & -1.15357 \\ \mathrm{H} & -0.06297 & 0.54436 & -2.02196 \\ \mathrm{~N} & 0.01933 & 1.04791 & 1.15082 \\ \mathrm{H} & -0.09614 & 2.05246 & 1.15357 \\ \mathrm{H} & -0.06297 & 0.54436 & 2.02196\end{array}$

$\begin{array}{lccc}\text { 1 planar conformer MP2/aug-cc-pVDZ T.E. }=-547.319544158 \text { a.u. }(-275.9 ;-1065 \mathrm{~cm}-1) \\ \mathrm{S} & 0.00000 & 0.00000 & 1.47985 \\ \mathrm{~N} & 0.00000 & 1.15002 & -0.92648 \\ \mathrm{C} & 0.00000 & 0.00000 & -0.19225 \\ \mathrm{H} & 0.00000 & 1.15614 & -1.93714 \\ \mathrm{H} & 0.00000 & 2.02580 & -0.42518 \\ \mathrm{~N} & 0.00000 & -1.15002 & -0.92648 \\ \mathrm{H} & 0.00000 & -1.15614 & -1.93714 \\ \mathrm{H} & 0.00000 & -2.02580 & -0.42518 \\ & & & \\ \mathbf{1} & \mathrm{TS} 1 \mathrm{conformer} \mathrm{MP} 2 / \text { aug-cc-pVDZ } & \text { T.E. }=-547.306608625 \text { a.u. }(-334.4 \mathrm{~cm}-1) \\ \mathrm{S} & -0.09362 & -1.47565 & 0.00000 \\ \mathrm{~N} & -1.06679 & 1.11306 & 0.00000 \\ \mathrm{C} & 0.03951 & 0.17651 & 0.00000 \\ \mathrm{H} & -1.65216 & 0.87353 & -0.80439 \\ \mathrm{H} & -1.65216 & 0.87353 & 0.80439 \\ \mathrm{~N} & 1.21240 & 0.84542 & 0.00000 \\ \mathrm{H} & 1.19994 & 1.85726 & 0.00000 \\ \mathrm{H} & 2.08441 & 0.33264 & 0.00000\end{array}$


1 TS2 conformer MP2/aug-cc-pVDZ T.E. $=-547.295848636$ a.u. $(-403.5 \mathrm{~cm}-1)$

$\begin{array}{lccc}\mathrm{S} & -0.06291 & 1.37053 & 0.00027 \\ \mathrm{~N} & -1.17827 & -1.09480 & 0.00068 \\ \mathrm{C} & 0.00958 & -0.27717 & -0.00037 \\ \mathrm{H} & -1.18776 & -1.70030 & -0.82297 \\ \mathrm{H} & -1.18395 & -1.70193 & 0.82319 \\ \mathrm{~N} & 1.18688 & -0.96691 & 0.00309 \\ \mathrm{H} & 1.20183 & -1.97770 & -0.01949 \\ \mathrm{H} & 2.05919 & -0.45346 & -0.00915\end{array}$

2 cis conformer MP2/aug-cc-pVDZ T.E. $=-586.502773780$ a.u.

$\begin{array}{llll}\mathrm{S} & 1.45529 & -0.68237 & -0.08186 \\ \mathrm{~N} & -1.11094 & -1.46855 & -0.05886 \\ \mathrm{~N} & -0.76482 & 0.80612 & 0.03747 \\ \mathrm{C} & -0.19341 & -0.42945 & -0.02055 \\ \mathrm{H} & -1.98275 & -1.33366 & 0.44484 \\ \mathrm{H} & -0.68882 & -2.38050 & 0.07306 \\ \mathrm{H} & -1.74934 & 0.86924 & -0.19681 \\ \mathrm{C} & 0.02905 & 2.02440 & -0.04546 \\ \mathrm{H} & 0.76749 & 2.03797 & 0.76633 \\ \mathrm{H} & 0.56890 & 2.08594 & -1.00273 \\ \mathrm{H} & -0.64943 & 2.88075 & 0.05979\end{array}$

2 trans conformer MP2/aug-cc-pVDZ T.E. $=-586.501661824$ a.u.

$\begin{array}{lrrr}\mathrm{S} & 0.54536 & 1.85487 & -0.05113 \\ \mathrm{~N} & 0.68468 & -0.80869 & -0.05421 \\ \mathrm{~N} & -1.44662 & 0.06698 & 0.02640 \\ \mathrm{C} & -0.09443 & 0.30616 & -0.03945 \\ \mathrm{C} & 0.19416 & -2.16953 & 0.09027 \\ \mathrm{H} & 1.67933 & -0.62348 & -0.04605 \\ \mathrm{H} & -1.82486 & -0.82041 & -0.28363 \\ \mathrm{H} & -2.02668 & 0.88308 & -0.11962 \\ \mathrm{H} & -0.40085 & -2.48271 & -0.78423 \\ \mathrm{H} & 1.05629 & -2.84239 & 0.16625 \\ \mathrm{H} & -0.41648 & -2.27729 & 1.00107\end{array}$

2 TS1 conformer MP2/aug-cc-pVDZ T.E. $=-586.486518199$ a.u. $(-140.8 \mathrm{~cm}-1)$

$\begin{array}{llrr}\mathrm{S} & 0.78140 & -1.35022 & -0.03496 \\ \mathrm{~N} & -1.72252 & -0.45405 & -0.17237 \\ \mathrm{~N} & -0.23339 & 1.17122 & 0.46575 \\ \mathrm{C} & -0.41839 & -0.21164 & 0.08391 \\ \mathrm{H} & -2.38414 & 0.30739 & -0.08447 \\ \mathrm{H} & -2.01941 & -1.38126 & -0.44887 \\ \mathrm{H} & 0.10357 & 1.15407 & 1.43129 \\ \mathrm{C} & 0.80048 & 1.83531 & -0.35675 \\ \mathrm{H} & 0.44963 & 1.86937 & -1.39744 \\ \mathrm{H} & 0.91492 & 2.86567 & 0.00626 \\ \mathrm{H} & 1.77164 & 1.31547 & -0.32468\end{array}$

2 TS2 conformer MP2/aug-cc-pVDZ T.E. $=-586.474498354$ a.u. $(-166.0 \mathrm{~cm}-1)$

$\begin{array}{llll}\mathrm{S} & 0.45164 & 1.67960 & -0.09126\end{array}$

$\mathrm{N} \quad-1.54245 \quad-0.06580 \quad-0.04066$

$\begin{array}{llll}\mathrm{N} & 0.57484 & -0.94801 & 0.53568\end{array}$

$\begin{array}{llll}\mathrm{C} & -0.21406 & 0.18720 & 0.13857\end{array}$

$\begin{array}{llll}\mathrm{H} & -1.92301 & -0.99467 & 0.08429\end{array}$ 


$\begin{array}{cccc}\mathrm{H} & -2.15015 & 0.68189 & -0.35032 \\ \mathrm{H} & 0.29676 & -1.24144 & 1.47368 \\ \mathrm{C} & 0.51909 & -2.07391 & -0.41012 \\ \mathrm{H} & -0.47768 & -2.53919 & -0.53732 \\ \mathrm{H} & 0.85951 & -1.71209 & -1.39040 \\ \mathrm{H} & 1.21856 & -2.84830 & -0.06788\end{array}$

3a cis conformer MP2/aug-cc-pVDZ T.E. $=-625.697091653$ a.u.

$\begin{array}{lrrr}\mathrm{S} & -1.31273 & -1.07793 & -0.10762 \\ \mathrm{~N} & 1.22335 & -1.80523 & 0.34909 \\ \mathrm{~N} & 0.93589 & 0.38013 & -0.31224 \\ \mathrm{C} & 0.33613 & -0.81165 & -0.03450 \\ \mathrm{H} & 2.15529 & -1.77715 & -0.05438 \\ \mathrm{H} & 0.80454 & -2.72785 & 0.37622 \\ \mathrm{H} & 1.89872 & 0.48672 & -0.00762 \\ \mathrm{C} & 0.18278 & 1.60483 & -0.57266 \\ \mathrm{C} & -0.30471 & 2.27644 & 0.71128 \\ \mathrm{H} & 0.84188 & 2.27380 & -1.14621 \\ \mathrm{H} & -0.66732 & 1.32646 & -1.20974 \\ \mathrm{H} & 0.54387 & 2.56262 & 1.35145 \\ \mathrm{H} & -0.87728 & 3.18527 & 0.47069 \\ \mathrm{H} & -0.95596 & 1.58653 & 1.26524\end{array}$

3a trans conformer MP2/aug-cc-pVDZ T.E. $=-625.694666369$ a.u.

$\begin{array}{lrrr}\mathrm{S} & -0.36815 & -2.18892 & -0.29582 \\ \mathrm{~N} & 1.48898 & -0.31739 & 0.15864 \\ \mathrm{~N} & -0.71191 & 0.39349 & 0.26374 \\ \mathrm{C} & 0.15363 & -0.63137 & 0.03847 \\ \mathrm{H} & 1.80251 & 0.63160 & -0.00624 \\ \mathrm{H} & 2.11461 & -1.05278 & -0.14510 \\ \mathrm{H} & -1.68746 & 0.13372 & 0.18085 \\ \mathrm{C} & -0.35420 & 1.76533 & 0.60293 \\ \mathrm{C} & -0.05021 & 2.63366 & -0.62312 \\ \mathrm{H} & -1.20005 & 2.19021 & 1.16259 \\ \mathrm{H} & 0.50054 & 1.74295 & 1.29796 \\ \mathrm{H} & -0.93086 & 2.68977 & -1.27869 \\ \mathrm{H} & 0.22168 & 3.65405 & -0.31230 \\ \mathrm{H} & 0.77978 & 2.21977 & -1.21553\end{array}$

3a TS1 conformer MP2/aug-cc-pVDZ T.E. $=-625.677167590$ a.u $(-130.6 \mathrm{~cm}-1)$

$\begin{array}{lrrr}\mathrm{S} & -1.30481 & 1.11687 & 0.20467 \\ \mathrm{~N} & 1.25397 & 1.82643 & 0.15404 \\ \mathrm{~N} & 0.94839 & -0.35436 & -0.47254 \\ \mathrm{C} & 0.31915 & 0.86959 & -0.04325 \\ \mathrm{H} & 2.22651 & 1.59509 & -0.00632 \\ \mathrm{H} & 0.97375 & 2.75187 & 0.45349 \\ \mathrm{H} & 0.60967 & -0.55139 & -1.41720 \\ \mathrm{C} & 0.62976 & -1.52097 & 0.39500 \\ \mathrm{C} & -0.59294 & -2.32579 & -0.04442 \\ \mathrm{H} & 1.52195 & -2.16698 & 0.40232 \\ \mathrm{H} & 0.49338 & -1.13741 & 1.41756 \\ \mathrm{H} & -0.45536 & -2.69551 & -1.07394 \\ \mathrm{H} & -0.72704 & -3.20085 & 0.61050 \\ \mathrm{H} & -1.49919 & -1.70782 & -0.00914\end{array}$

3a TS2 conformer MP2/aug-cc-pVDZ T.E. $=-625.665549401$ a.u $(-195.4 \mathrm{~cm}-1)$ 


$\begin{array}{lrrr}\mathrm{S} & 0.29990 & 2.17879 & 0.17259 \\ \mathrm{~N} & -1.49448 & 0.25063 & -0.09588 \\ \mathrm{~N} & 0.74402 & -0.38502 & -0.54358 \\ \mathrm{C} & -0.18571 & 0.63801 & -0.17668 \\ \mathrm{H} & -1.78964 & -0.63565 & -0.48275 \\ \mathrm{H} & -2.19177 & 0.95446 & 0.11556 \\ \mathrm{H} & 0.68460 & -0.61017 & -1.53605 \\ \mathrm{C} & 0.82479 & -1.57373 & 0.32076 \\ \mathrm{C} & -0.25090 & -2.65843 & 0.16001 \\ \mathrm{H} & 1.81102 & -2.02454 & 0.12723 \\ \mathrm{H} & 0.84513 & -1.20391 & 1.35786 \\ \mathrm{H} & -0.35688 & -2.94992 & -0.89755 \\ \mathrm{H} & 0.04085 & -3.55790 & 0.72472 \\ \mathrm{H} & -1.23024 & -2.33975 & 0.54533\end{array}$

3b cis conformer MP2/aug-cc-pVDZ T.E. $=-625.696530740$ a.u.

$\begin{array}{lrrr}\mathrm{S} & -1.35273 & -1.01809 & 0.27566 \\ \mathrm{~N} & 1.10957 & -1.92958 & -0.25207 \\ \mathrm{~N} & 0.97209 & 0.31732 & 0.23173 \\ \mathrm{C} & 0.29857 & -0.85174 & 0.06489 \\ \mathrm{H} & 1.95255 & -1.74478 & -0.78727 \\ \mathrm{H} & 0.59368 & -2.75127 & -0.54400 \\ \mathrm{H} & 1.98529 & 0.28719 & 0.21269 \\ \mathrm{C} & 0.32365 & 1.57283 & 0.60358 \\ \mathrm{C} & -0.26742 & 2.29892 & -0.60433 \\ \mathrm{H} & -0.46426 & 1.33670 & 1.33329 \\ \mathrm{H} & 1.08553 & 2.19163 & 1.10066 \\ \mathrm{H} & -1.01078 & 1.65575 & -1.09467 \\ \mathrm{H} & -0.76374 & 3.22726 & -0.28232 \\ \mathrm{H} & 0.51922 & 2.55714 & -1.32888\end{array}$

3b trans conformer MP2/aug-cc-pVDZ T.E. $=-625.695082892$ a.u.

$\begin{array}{lrrr}\mathrm{S} & -0.43036 & -2.15920 & 0.06013 \\ \mathrm{~N} & 1.51575 & -0.32598 & 0.04729 \\ \mathrm{~N} & -0.57017 & 0.40758 & -0.65071 \\ \mathrm{C} & 0.19610 & -0.62870 & -0.19991 \\ \mathrm{H} & 1.94494 & 0.46033 & -0.42728 \\ \mathrm{H} & 2.10535 & -1.13678 & 0.18809 \\ \mathrm{H} & -1.55413 & 0.16784 & -0.70947 \\ \mathrm{C} & -0.21587 & 1.82183 & -0.54873 \\ \mathrm{C} & -0.23614 & 2.34516 & 0.89026 \\ \mathrm{H} & 0.77007 & 1.98568 & -1.01350 \\ \mathrm{H} & -0.93558 & 2.37170 & -1.17233 \\ \mathrm{H} & 0.46734 & 1.77964 & 1.51722 \\ \mathrm{H} & 0.04470 & 3.40926 & 0.91525 \\ \mathrm{H} & -1.24337 & 2.23998 & 1.31867\end{array}$

3b TS1 conformer MP2/aug-cc-pVDZ T.E. $=-625.681986392$ a.u $(-89.9 \mathrm{~cm}-1)$

$\begin{array}{llll}\mathrm{S} & 0.82001 & 1.56925 & -0.31953 \\ \mathrm{~N} & -1.72572 & 0.81160 & -0.14459 \\ \mathrm{~N} & -0.30094 & -0.59975 & 0.97500 \\ \mathrm{C} & -0.43040 & 0.59599 & 0.17009 \\ \mathrm{H} & -2.42154 & 0.15681 & 0.19109 \\ \mathrm{H} & -1.98481 & 1.62231 & -0.69254 \\ \mathrm{H} & 0.05124 & -0.28868 & 1.88402 \\ \mathrm{C} & 0.69373 & -1.54367 & 0.40723\end{array}$




$\begin{array}{lrrr}\mathrm{C} & 0.18506 & -2.10651 & -0.91470 \\ \mathrm{H} & 1.67103 & -1.05062 & 0.26219 \\ \mathrm{H} & 0.81333 & -2.34948 & 1.14704 \\ \mathrm{H} & 0.06163 & -1.30279 & -1.65664 \\ \mathrm{H} & 0.90460 & -2.83200 & -1.32228 \\ \mathrm{H} & -0.78233 & -2.61105 & -0.77412\end{array}$

3b TS2 conformer MP2/aug-cc-pVDZ T.E.= -625.669900567 a.u (-94.3 cm-1)

$\begin{array}{llcc}\mathrm{S} & 0.69860 & 1.80141 & 0.01785 \\ \mathrm{~N} & -1.55456 & 0.51868 & 0.56470 \\ \mathrm{~N} & -0.14734 & -0.56128 & -0.99831 \\ \mathrm{C} & -0.38187 & 0.56342 & -0.13037 \\ \mathrm{H} & -2.19070 & -0.26262 & 0.47130 \\ \mathrm{H} & -1.77451 & 1.26484 & 1.21225 \\ \mathrm{H} & -0.86255 & -0.56997 & -1.72894 \\ \mathrm{C} & -0.11466 & -1.86174 & -0.29388 \\ \mathrm{C} & 1.02843 & -1.88386 & 0.71554 \\ \mathrm{H} & -1.06703 & -2.11183 & 0.21845 \\ \mathrm{H} & 0.04633 & -2.62767 & -1.06725 \\ \mathrm{H} & 0.87936 & -1.12582 & 1.49884 \\ \mathrm{H} & 1.08683 & -2.87047 & 1.19880 \\ \mathrm{H} & 1.98137 & -1.67106 & 0.21141\end{array}$

3c cis conformer MP2/aug-cc-pVDZ T.E. $=-625.696163571$ a.u.

$\begin{array}{lrrr}\mathrm{S} & -1.10615 & 1.55533 & -0.14996 \\ \mathrm{~N} & 1.57463 & 1.54899 & -0.03381 \\ \mathrm{~N} & 0.57120 & -0.52367 & 0.02242 \\ \mathrm{C} & 0.39133 & 0.82557 & -0.03858 \\ \mathrm{H} & 2.35158 & 1.16012 & 0.49261 \\ \mathrm{H} & 1.43529 & 2.54410 & 0.09933 \\ \mathrm{H} & 1.50191 & -0.87499 & -0.18332 \\ \mathrm{C} & -0.53602 & -1.46647 & -0.11012 \\ \mathrm{C} & -0.01778 & -2.89091 & 0.05506 \\ \mathrm{H} & -1.28087 & -1.22140 & 0.66003 \\ \mathrm{H} & -1.02706 & -1.32828 & -1.08766 \\ \mathrm{H} & 0.44191 & -3.03041 & 1.04485 \\ \mathrm{H} & -0.84762 & -3.60456 & -0.04337 \\ \mathrm{H} & 0.72742 & -3.13625 & -0.71840\end{array}$

3c trans conformer MP2/aug-cc-pVDZ T.E. $=-625.695552754$ a.u.

$\begin{array}{lrrr}\mathrm{S} & 0.68365 & -2.15132 & 0.11177 \\ \mathrm{~N} & -1.58619 & -0.74555 & -0.08926 \\ \mathrm{~N} & 0.34472 & 0.49511 & 0.12569 \\ \mathrm{C} & -0.21986 & -0.74036 & 0.06123 \\ \mathrm{H} & -2.13409 & 0.05954 & 0.19045 \\ \mathrm{H} & -2.02040 & -1.65293 & 0.01956 \\ \mathrm{H} & 1.35671 & 0.49206 & 0.18290 \\ \mathrm{C} & -0.36097 & 1.75988 & -0.03869 \\ \mathrm{C} & 0.64361 & 2.90682 & -0.06827 \\ \mathrm{H} & -0.94400 & 1.73683 & -0.97540 \\ \mathrm{H} & -1.06749 & 1.90912 & 0.79793 \\ \mathrm{H} & 1.34120 & 2.78741 & -0.91033 \\ \mathrm{H} & 0.12164 & 3.86637 & -0.18619 \\ \mathrm{H} & 1.22033 & 2.94120 & 0.86795\end{array}$

3c TS1 conformer MP2/aug-cc-pVDZ T.E.= -625.680470683 a.u $(-136.8 \mathrm{~cm}-1)$ 


$\begin{array}{lccc}\mathrm{S} & 1.02632 & 1.66028 & -0.03499 \\ \mathrm{~N} & -1.61483 & 1.34818 & -0.15420 \\ \mathrm{~N} & -0.51955 & -0.58587 & 0.41751 \\ \mathrm{C} & -0.39546 & 0.81326 & 0.07598 \\ \mathrm{H} & -2.42943 & 0.75190 & -0.07409 \\ \mathrm{H} & -1.69961 & 2.32688 & -0.39784 \\ \mathrm{H} & -0.19689 & -0.67289 & 1.38584 \\ \mathrm{C} & 0.35167 & -1.44757 & -0.41523 \\ \mathrm{C} & 0.20964 & -2.89682 & 0.03449 \\ \mathrm{H} & 0.01621 & -1.33164 & -1.45753 \\ \mathrm{H} & 1.40379 & -1.11876 & -0.36494 \\ \mathrm{H} & -0.83549 & -3.22950 & -0.03964 \\ \mathrm{H} & 0.83521 & -3.55173 & -0.58981 \\ \mathrm{H} & 0.53822 & -3.01452 & 1.07948\end{array}$

3c TS2 conformer MP2/aug-cc-pVDZ T.E. $=-625.668853107$ a.u $(-157.8 \mathrm{~cm}-1)$

$\begin{array}{lccc}\mathrm{S} & 0.75899 & 2.02200 & 0.10702 \\ \mathrm{~N} & -1.61511 & 0.84572 & 0.03666 \\ \mathrm{~N} & 0.21056 & -0.55165 & -0.51577 \\ \mathrm{C} & -0.26465 & 0.74899 & -0.12900 \\ \mathrm{H} & -2.21737 & 0.04167 & -0.08202 \\ \mathrm{H} & -2.01359 & 1.72494 & 0.34091 \\ \mathrm{H} & -0.12434 & -0.77065 & -1.45727 \\ \mathrm{C} & -0.14177 & -1.63052 & 0.42571 \\ \mathrm{C} & 0.55985 & -2.91838 & 0.00853 \\ \mathrm{H} & 0.20858 & -1.30695 & 1.41824 \\ \mathrm{H} & -1.23310 & -1.81375 & 0.50724 \\ \mathrm{H} & 1.64719 & -2.76430 & -0.01797 \\ \mathrm{H} & 0.32760 & -3.72960 & 0.71444 \\ \mathrm{H} & 0.22935 & -3.23571 & -0.99323\end{array}$

4a cis conformer MP2/aug-cc-pVDZ T.E.= -664.893584147 a.u.

$\begin{array}{lccc}\mathrm{S} & 1.08153 & -1.67640 & 0.39728 \\ \mathrm{~N} & -1.47647 & -1.91683 & -0.35473 \\ \mathrm{~N} & -0.75815 & 0.24176 & -0.00611 \\ \mathrm{C} & -0.43244 & -1.08050 & 0.01019 \\ \mathrm{H} & -2.41814 & -1.61184 & -0.12575 \\ \mathrm{H} & -1.29247 & -2.89482 & -0.16185 \\ \mathrm{H} & -1.62540 & 0.49430 & -0.47355 \\ \mathrm{C} & 0.22004 & 1.31674 & 0.17649 \\ \mathrm{C} & 1.00377 & 1.56564 & -1.11418 \\ \mathrm{C} & -0.51663 & 2.56540 & 0.65721 \\ \mathrm{H} & 0.90888 & 0.96012 & 0.95623 \\ \mathrm{H} & 0.32791 & 1.91878 & -1.91015 \\ \mathrm{H} & 1.77724 & 2.33198 & -0.95101 \\ \mathrm{H} & 1.49294 & 0.63783 & -1.44101 \\ \mathrm{H} & -1.24925 & 2.90149 & -0.09606 \\ \mathrm{H} & -1.04554 & 2.37338 & 1.60199 \\ \mathrm{H} & 0.19782 & 3.38619 & 0.81553\end{array}$

4a trans conformer MP2/aug-cc-pVDZ T.E. $=-664.891450424$ a.u.

$\begin{array}{lrrr}\mathrm{S} & -0.44448 & -2.47760 & -0.38023 \\ \mathrm{~N} & 1.44957 & -0.88575 & 0.63586 \\ \mathrm{~N} & -0.46536 & 0.17413 & -0.12020 \\ \mathrm{C} & 0.21127 & -0.99358 & 0.04479 \\ \mathrm{H} & 1.97185 & -0.02064 & 0.56910\end{array}$




$\begin{array}{lrrr}\mathrm{H} & 1.99122 & -1.74058 & 0.62399 \\ \mathrm{H} & -1.38285 & 0.06289 & -0.53896 \\ \mathrm{C} & -0.01040 & 1.51226 & 0.25310 \\ \mathrm{C} & 0.89630 & 2.12097 & -0.82502 \\ \mathrm{C} & -1.24045 & 2.38505 & 0.50106 \\ \mathrm{H} & 0.54426 & 1.41784 & 1.20259 \\ \mathrm{H} & 0.32321 & 2.26649 & -1.75349 \\ \mathrm{H} & 1.28745 & 3.09641 & -0.49638 \\ \mathrm{H} & 1.74890 & 1.46624 & -1.05999 \\ \mathrm{H} & -1.84557 & 2.45744 & -0.41741 \\ \mathrm{H} & -1.86377 & 1.96497 & 1.30305 \\ \mathrm{H} & -0.93548 & 3.40208 & 0.78646\end{array}$

4a TS1 conformer MP2/aug-cc-pVDZ T.E.= -664.873861159 a.u (-123.7 cm-1)

$\begin{array}{lrrr}\mathrm{S} & -0.85642 & 1.89979 & 0.37454 \\ \mathrm{~N} & 1.76519 & 1.56474 & 0.11300 \\ \mathrm{~N} & 0.58472 & -0.27528 & -0.56882 \\ \mathrm{C} & 0.51842 & 1.06213 & -0.03525 \\ \mathrm{H} & 2.55469 & 0.99110 & -0.15690 \\ \mathrm{H} & 1.89166 & 2.50448 & 0.46755 \\ \mathrm{H} & 0.13131 & -0.25509 & -1.48598 \\ \mathrm{C} & -0.11307 & -1.29263 & 0.27482 \\ \mathrm{C} & -1.56191 & -1.51830 & -0.16196 \\ \mathrm{C} & 0.69535 & -2.58934 & 0.22437 \\ \mathrm{H} & -0.10290 & -0.89737 & 1.30367 \\ \mathrm{H} & -1.58364 & -1.88848 & -1.20176 \\ \mathrm{H} & -2.04537 & -2.27534 & 0.47484 \\ \mathrm{H} & -2.13502 & -0.58382 & -0.10428 \\ \mathrm{H} & 0.73767 & -2.97133 & -0.80814 \\ \mathrm{H} & 1.72450 & -2.41942 & 0.57079 \\ \mathrm{H} & 0.22899 & -3.35989 & 0.85831\end{array}$

4a TS2 conformer MP2/aug-cc-pVDZ T.E. $=-664.862081611$ a.u $(-170.0 \mathrm{~cm}-1)$

$\begin{array}{lrrr}\mathrm{S} & -0.71689 & -2.33759 & -0.01059 \\ \mathrm{~N} & 1.57276 & -1.01813 & 0.14127 \\ \mathrm{~N} & -0.25923 & 0.23878 & -0.67660 \\ \mathrm{C} & 0.24894 & -1.00918 & -0.19418 \\ \mathrm{H} & 2.16677 & -0.22803 & -0.06979 \\ \mathrm{H} & 1.98458 & -1.87523 & 0.48911 \\ \mathrm{H} & 0.11081 & 0.45260 & -1.60394 \\ \mathrm{C} & -0.15094 & 1.39078 & 0.25213 \\ \mathrm{C} & 1.05366 & 2.29847 & -0.02829 \\ \mathrm{C} & -1.45478 & 2.18885 & 0.17605 \\ \mathrm{H} & -0.05886 & 0.96064 & 1.26336 \\ \mathrm{H} & 1.00492 & 2.67870 & -1.06233 \\ \mathrm{H} & 1.05365 & 3.16623 & 0.64937 \\ \mathrm{H} & 2.01662 & 1.78145 & 0.10356 \\ \mathrm{H} & -1.58972 & 2.59436 & -0.83956 \\ \mathrm{H} & -2.30769 & 1.53450 & 0.39997 \\ \mathrm{H} & -1.44499 & 3.02961 & 0.88773\end{array}$

4b cis conformer MP2/aug-cc-pVDZ T.E. $=-664.893121939$ a.u.

$\begin{array}{llll}\mathrm{S} & 1.02655 & 1.66402 & 0.58602 \\ \mathrm{~N} & -1.49121 & 1.93912 & -0.28471 \\ \mathrm{~N} & -0.75013 & -0.23118 & -0.08383 \\ \mathrm{C} & -0.44622 & 1.08756 & 0.03921\end{array}$




$\begin{array}{rrrr}\mathrm{H} & -2.14475 & 1.63074 & -0.99830 \\ \mathrm{H} & -1.21110 & 2.91012 & -0.35901 \\ \mathrm{H} & -1.71521 & -0.47849 & -0.28103 \\ \mathrm{C} & 0.17563 & -1.31800 & 0.24580 \\ \mathrm{C} & -0.64344 & -2.55537 & 0.60697 \\ \mathrm{C} & 1.13504 & -1.57951 & -0.91638 \\ \mathrm{H} & 0.75239 & -0.97834 & 1.12023 \\ \mathrm{H} & -1.26213 & -2.87696 & -0.24779 \\ \mathrm{H} & 0.02774 & -3.38696 & 0.86473 \\ \mathrm{H} & -1.29990 & -2.36060 & 1.46823 \\ \mathrm{H} & 0.57838 & -1.93504 & -1.79812 \\ \mathrm{H} & 1.67105 & -0.65639 & -1.17535 \\ \mathrm{H} & 1.87371 & -2.34613 & -0.63510\end{array}$

4b trans conformer MP2/aug-cc-pVDZ T.E. $=-664.891931849$ a.u.

$\begin{array}{lrrr}\mathrm{S} & -0.65005 & -2.43060 & 0.11880 \\ \mathrm{~N} & 1.45028 & -0.93315 & -0.58328 \\ \mathrm{~N} & -0.53535 & 0.21064 & -0.23159 \\ \mathrm{C} & 0.11546 & -0.98839 & -0.25365 \\ \mathrm{H} & 1.80077 & -0.14666 & -1.11828 \\ \mathrm{H} & 1.86420 & -1.83426 & -0.78685 \\ \mathrm{H} & -1.48564 & 0.13517 & 0.11985 \\ \mathrm{C} & 0.09691 & 1.53194 & -0.18606 \\ \mathrm{C} & -0.98624 & 2.58003 & -0.43776 \\ \mathrm{C} & 0.81748 & 1.76536 & 1.14663 \\ \mathrm{H} & 0.82273 & 1.59207 & -1.01611 \\ \mathrm{H} & -1.74990 & 2.53248 & 0.35557 \\ \mathrm{H} & -0.54963 & 3.58886 & -0.42685 \\ \mathrm{H} & -1.47566 & 2.41575 & -1.40834 \\ \mathrm{H} & 0.08484 & 1.76323 & 1.96843 \\ \mathrm{H} & 1.55606 & 0.97463 & 1.33848 \\ \mathrm{H} & 1.33432 & 2.73764 & 1.14018\end{array}$

$\begin{array}{lccc}\text { 4b TS1 } & \text { conformer MP2/aug-cc-pVDZ T.E. }=-664.879138901 \text { a.u }(-92.1 \mathrm{~cm}-1) \\ \mathrm{S} & -0.96671 & 1.84685 & 0.30586 \\ \mathrm{~N} & 1.60199 & 1.49122 & -0.29272 \\ \mathrm{~N} & 0.31330 & -0.34397 & -0.78801 \\ \mathrm{C} & 0.34269 & 1.00386 & -0.26517 \\ \mathrm{H} & 2.34329 & 0.89869 & -0.64645 \\ \mathrm{H} & 1.78620 & 2.43470 & 0.02451 \\ \mathrm{H} & -0.23857 & -0.30007 & -1.65003 \\ \mathrm{C} & -0.36754 & -1.29768 & 0.12669 \\ \mathrm{C} & -0.51322 & -2.62582 & -0.60987 \\ \mathrm{C} & 0.46939 & -1.44115 & 1.39436 \\ \mathrm{H} & -1.36747 & -0.90981 & 0.39557 \\ \mathrm{H} & 0.47613 & -3.01966 & -0.88877 \\ \mathrm{H} & -1.01920 & -3.36202 & 0.03251 \\ \mathrm{H} & -1.11362 & -2.50819 & -1.52616 \\ \mathrm{H} & 1.46717 & -1.83608 & 1.14594 \\ \mathrm{H} & 0.58630 & -0.47243 & 1.90323 \\ \mathrm{H} & -0.02027 & -2.13303 & 2.09618 \\ & & & \\ \mathbf{4 b} \text { TS2 } & \text { conformer MP2/aug-cc-pVDZ T.E. }=-664.867445376 \text { a.u }(-90.5 \mathrm{~cm}-1) \\ \mathrm{S} & 0.73327 & -2.17092 & 0.12986 \\ \mathrm{~N} & -1.59609 & -1.04602 & -0.44449 \\ \mathrm{~N} & -0.10346 & 0.32212 & 0.77384\end{array}$




$\begin{array}{lrrr}\mathrm{C} & -0.37020 & -0.94432 & 0.14456 \\ \mathrm{H} & -2.24217 & -0.26726 & -0.44618 \\ \mathrm{H} & -1.84521 & -1.90181 & -0.92425 \\ \mathrm{H} & -0.76183 & 0.45172 & 1.54712 \\ \mathrm{C} & -0.16390 & 1.48753 & -0.14011 \\ \mathrm{C} & 0.12807 & 2.73589 & 0.68839 \\ \mathrm{C} & 0.86336 & 1.29664 & -1.25314 \\ \mathrm{H} & -1.16722 & 1.60548 & -0.60504 \\ \mathrm{H} & 1.13076 & 2.65903 & 1.13408 \\ \mathrm{H} & 0.08340 & 3.63473 & 0.05519 \\ \mathrm{H} & -0.60626 & 2.85547 & 1.50114 \\ \mathrm{H} & 1.86487 & 1.17422 & -0.81437 \\ \mathrm{H} & 0.64360 & 0.40449 & -1.85782 \\ \mathrm{H} & 0.86237 & 2.17318 & -1.91857\end{array}$

$4 \mathbf{c}$ cis conformer MP2/aug-cc-pVDZ T.E. $=-664.888075283$ a.u.

$\begin{array}{lrrr}\mathrm{S} & -1.35669 & 1.20569 & -0.15230 \\ \mathrm{~N} & 1.09301 & 2.24298 & 0.03731 \\ \mathrm{~N} & 1.03316 & -0.04747 & 0.10353 \\ \mathrm{C} & 0.30441 & 1.09808 & 0.01453 \\ \mathrm{H} & 1.93444 & 2.21500 & 0.60622 \\ \mathrm{H} & 0.55025 & 3.09328 & 0.13892 \\ \mathrm{H} & 2.03575 & 0.06645 & -0.00546 \\ \mathrm{C} & 0.54175 & -1.43612 & 0.02550 \\ \mathrm{C} & -0.39311 & -1.78669 & 1.18653 \\ \mathrm{C} & -0.06759 & -1.76350 & -1.34195 \\ \mathrm{H} & 1.45987 & -2.03535 & 0.14387 \\ \mathrm{H} & -1.34669 & -1.25333 & 1.09367 \\ \mathrm{H} & -0.58674 & -2.87037 & 1.17658 \\ \mathrm{H} & 0.07003 & -1.52382 & 2.14885 \\ \mathrm{H} & -0.99048 & -1.19069 & -1.49637 \\ \mathrm{H} & 0.63940 & -1.52357 & -2.15009 \\ \mathrm{H} & -0.29967 & -2.83871 & -1.39031\end{array}$

4c trans conformer MP2/aug-cc-pVDZ T.E. $=-664.886684373$ a.u.

\begin{tabular}{lccc}
$\mathrm{S}$ & 0.25222 & 2.48069 & -0.20351 \\
$\mathrm{~N}$ & -1.31229 & 0.50581 & 0.68960 \\
$\mathrm{~N}$ & 0.38855 & -0.12159 & -0.77278 \\
$\mathrm{C}$ & -0.24594 & 0.88743 & -0.09600 \\
$\mathrm{H}$ & -1.84988 & -0.30152 & 0.39732 \\
$\mathrm{H}$ & -1.85563 & 1.28861 & 1.03419 \\
$\mathrm{H}$ & 1.20286 & 0.23933 & -1.26099 \\
$\mathrm{C}$ & 0.49319 & -1.53659 & -0.35509 \\
$\mathrm{C}$ & 1.05970 & -1.67382 & 1.06290 \\
$\mathrm{C}$ & -0.80644 & -2.33309 & -0.53682 \\
$\mathrm{H}$ & 1.22305 & -1.95371 & -1.06722 \\
$\mathrm{H}$ & 0.37561 & -1.22216 & 1.79550 \\
$\mathrm{H}$ & 1.19213 & -2.73721 & 1.31599 \\
$\mathrm{H}$ & 2.03436 & -1.17097 & 1.14099 \\
$\mathrm{H}$ & -1.50422 & -2.18887 & 0.30237 \\
$\mathrm{H}$ & -1.30496 & -2.06644 & -1.47994 \\
$\mathrm{H}$ & -0.57163 & -3.40781 & -0.56233 \\
& \multicolumn{3}{c}{} \\
$\mathbf{4 c}$ TS1 conformer MP2/aug-cc-pVDZ T.E. $=-664.873179382$ a.u $(-123.9 \mathrm{~cm}-1)$ \\
$\mathrm{S}$ & 1.13933 & -1.45610 & 0.39039 \\
$\mathrm{~N}$ & -1.44157 & -1.80204 & -0.09227
\end{tabular}




$\begin{array}{rrrr}\mathrm{N} & -0.70212 & 0.21308 & -0.87232 \\ \mathrm{C} & -0.35027 & -1.00694 & -0.19327 \\ \mathrm{H} & -2.32799 & -1.45819 & -0.44041 \\ \mathrm{H} & -1.35697 & -2.72209 & 0.32158 \\ \mathrm{H} & -0.30996 & 0.16288 & -1.81539 \\ \mathrm{C} & -0.28873 & 1.49652 & -0.24008 \\ \mathrm{C} & -0.75009 & 1.51923 & 1.21651 \\ \mathrm{C} & 1.19521 & 1.85753 & -0.37875 \\ \mathrm{H} & -0.87339 & 2.25197 & -0.79291 \\ \mathrm{H} & -0.18762 & 0.78776 & 1.81529 \\ \mathrm{H} & -0.57716 & 2.51768 & 1.64483 \\ \mathrm{H} & -1.82407 & 1.29176 & 1.28992 \\ \mathrm{H} & 1.82436 & 1.20138 & 0.23512 \\ \mathrm{H} & 1.52364 & 1.76925 & -1.42681 \\ \mathrm{H} & 1.34625 & 2.90252 & -0.06334\end{array}$

4c TS2 conformer MP2/aug-cc-pVDZ T.E. $=-664.863075121$ a.u $(-164.5 \mathrm{~cm}-1)$

$\begin{array}{lccc}\mathrm{S} & -0.42576 & 2.29199 & -0.04247 \\ \mathrm{~N} & 1.50164 & 0.54491 & -0.52527 \\ \mathrm{~N} & -0.16966 & -0.18742 & 0.99233 \\ \mathrm{C} & 0.34781 & 0.84355 & 0.14467 \\ \mathrm{H} & 2.05190 & -0.26105 & -0.26031 \\ \mathrm{H} & 1.92849 & 1.27040 & -1.08919 \\ \mathrm{H} & 0.37287 & -0.26022 & 1.85294 \\ \mathrm{C} & -0.47681 & -1.50659 & 0.39485 \\ \mathrm{C} & -1.37020 & -1.29886 & -0.82809 \\ \mathrm{C} & 0.72049 & -2.41454 & 0.06109 \\ \mathrm{H} & -1.06900 & -2.01697 & 1.17328 \\ \mathrm{H} & -0.82361 & -0.76910 & -1.62412 \\ \mathrm{H} & -1.69340 & -2.27244 & -1.22635 \\ \mathrm{H} & -2.25645 & -0.70619 & -0.56317 \\ \mathrm{H} & 1.24015 & -2.08607 & -0.85228 \\ \mathrm{H} & 1.43875 & -2.45297 & 0.89655 \\ \mathrm{H} & 0.36690 & -3.44193 & -0.11973\end{array}$

5 cis conformer MP2/aug-cc-pVDZ T.E. $=-704.086858530$ a.u.

$\begin{array}{lccc}\mathrm{S} & 1.04960 & -1.89373 & -0.08277 \\ \mathrm{~N} & -1.60516 & -2.10258 & 0.01316 \\ \mathrm{~N} & -0.83895 & 0.05528 & 0.05002 \\ \mathrm{C} & -0.49684 & -1.26154 & 0.00939 \\ \mathrm{H} & -2.41602 & -1.79770 & 0.54443 \\ \mathrm{H} & -1.35947 & -3.07640 & 0.15218 \\ \mathrm{H} & -1.82658 & 0.24610 & -0.09724 \\ \mathrm{C} & 0.03986 & 1.24374 & -0.02817 \\ \mathrm{C} & 0.79188 & 1.27892 & -1.36537 \\ \mathrm{C} & -0.91007 & 2.44423 & 0.06063 \\ \mathrm{C} & 1.00919 & 1.27563 & 1.15954 \\ \mathrm{H} & 0.07882 & 1.26999 & -2.20435 \\ \mathrm{H} & 1.45812 & 0.41223 & -1.45671 \\ \mathrm{H} & 1.39186 & 2.20051 & -1.42537 \\ \mathrm{H} & -1.62952 & 2.43977 & -0.77519 \\ \mathrm{H} & -0.33402 & 3.37884 & 0.00605 \\ \mathrm{H} & -1.46579 & 2.43468 & 1.01189 \\ \mathrm{H} & 0.45089 & 1.22973 & 2.10700 \\ \mathrm{H} & 1.58108 & 2.21633 & 1.13365 \\ \mathrm{H} & 1.70858 & 0.43229 & 1.11818\end{array}$


5 trans conformer MP2/aug-cc-pVDZ T.E. $=-704.085400023$ a.u.

$\begin{array}{lrrr}\mathrm{S} & -0.58596 & -2.64660 & -0.11995 \\ \mathrm{~N} & 1.55726 & -1.06382 & 0.07732 \\ \mathrm{~N} & -0.44795 & -0.00978 & -0.48597 \\ \mathrm{C} & 0.20878 & -1.17568 & -0.18275 \\ \mathrm{H} & 2.06910 & -0.32943 & -0.39677 \\ \mathrm{H} & 2.02887 & -1.95940 & 0.12772 \\ \mathrm{H} & -1.44271 & -0.19565 & -0.59112 \\ \mathrm{C} & -0.14121 & 1.35508 & 0.01504 \\ \mathrm{C} & 1.12292 & 1.93904 & -0.63729 \\ \mathrm{C} & -1.34367 & 2.20569 & -0.41319 \\ \mathrm{C} & -0.01652 & 1.35049 & 1.54500 \\ \mathrm{H} & 1.12998 & 1.73572 & -1.71852 \\ \mathrm{H} & 2.04812 & 1.55437 & -0.18350 \\ \mathrm{H} & 1.13685 & 3.02979 & -0.48954 \\ \mathrm{H} & -1.43546 & 2.22208 & -1.51028 \\ \mathrm{H} & -1.22119 & 3.23801 & -0.05494 \\ \mathrm{H} & -2.27626 & 1.80398 & 0.01437 \\ \mathrm{H} & -0.95272 & 0.99628 & 2.00222 \\ \mathrm{H} & 0.19062 & 2.36876 & 1.91036 \\ \mathrm{H} & 0.80031 & 0.69002 & 1.86856\end{array}$

5 TS1 conformer MP2/aug-cc-pVDZ T.E.= -704.071927963 a.u (-126.3 cm-1)

$\begin{array}{lrrr}\mathrm{S} & -0.89149 & -2.03858 & -0.16426 \\ \mathrm{~N} & 1.72999 & -1.76533 & 0.07242 \\ \mathrm{~N} & 0.63572 & 0.14440 & 0.67190 \\ \mathrm{C} & 0.50223 & -1.20601 & 0.19142 \\ \mathrm{H} & 2.54447 & -1.19687 & 0.26958 \\ \mathrm{H} & 1.81614 & -2.73288 & -0.21304 \\ \mathrm{H} & 0.36005 & 0.15008 & 1.65772 \\ \mathrm{C} & -0.09358 & 1.23694 & -0.03885 \\ \mathrm{C} & -1.60381 & 1.26022 & 0.24120 \\ \mathrm{C} & 0.53845 & 2.53141 & 0.48856 \\ \mathrm{C} & 0.18010 & 1.10416 & -1.53881 \\ \mathrm{H} & -1.79101 & 1.31514 & 1.32653 \\ \mathrm{H} & -2.09519 & 0.36078 & -0.14815 \\ \mathrm{H} & -2.05459 & 2.15002 & -0.22707 \\ \mathrm{H} & 0.40797 & 2.60953 & 1.58056 \\ \mathrm{H} & 0.05653 & 3.40675 & 0.02606 \\ \mathrm{H} & 1.61523 & 2.55300 & 0.26574 \\ \mathrm{H} & 1.26413 & 1.06981 & -1.72936 \\ \mathrm{H} & -0.23753 & 1.97467 & -2.06703 \\ \mathrm{H} & -0.28431 & 0.19652 & -1.94996\end{array}$

5 TS2 conformer MP2/aug-cc-pVDZ T.E.= -704.061889655 a.u (-197.4 cm-1)

$\begin{array}{lccc}\mathrm{S} & 0.63401 & 2.48895 & 0.07717 \\ \mathrm{~N} & -1.62795 & 1.12258 & -0.03984 \\ \mathrm{~N} & 0.30744 & -0.05746 & -0.74614 \\ \mathrm{C} & -0.27778 & 1.14967 & -0.25252 \\ \mathrm{H} & -2.18940 & 0.37789 & -0.43087 \\ \mathrm{H} & -2.08129 & 1.98018 & 0.25287 \\ \mathrm{H} & 0.13131 & -0.17564 & -1.74453 \\ \mathrm{C} & 0.15597 & -1.32119 & 0.01708 \\ \mathrm{C} & -1.24687 & -1.96009 & -0.03920 \\ \mathrm{C} & 1.16394 & -2.28893 & -0.61571\end{array}$




$\begin{array}{cccc}\mathrm{C} & 0.54473 & -1.03629 & 1.47055 \\ \mathrm{H} & -1.60340 & -2.02807 & -1.08069 \\ \mathrm{H} & -1.97825 & -1.39967 & 0.56175 \\ \mathrm{H} & -1.21192 & -2.98264 & 0.36886 \\ \mathrm{H} & 0.90661 & -2.48183 & -1.67085 \\ \mathrm{H} & 1.15948 & -3.25294 & -0.08409 \\ \mathrm{H} & 2.17380 & -1.85707 & -0.57767 \\ \mathrm{H} & 1.55674 & -0.60922 & 1.51736 \\ \mathrm{H} & 0.52003 & -1.97136 & 2.05115 \\ \mathrm{H} & -0.15401 & -0.32501 & 1.93726\end{array}$

6 trans conformer MP2/aug-cc-pVDZ T.E. $=-777.703065323$ a.u.

$\begin{array}{lrrr}\mathrm{S} & 0.29693 & -3.39679 & -0.15525 \\ \mathrm{~N} & -1.25429 & -1.45623 & 0.83773 \\ \mathrm{~N} & 0.53041 & -0.76544 & -0.47576 \\ \mathrm{C} & -0.16242 & -1.80813 & 0.09313 \\ \mathrm{H} & -1.35013 & -0.50617 & 1.18147 \\ \mathrm{H} & -1.66472 & -2.20503 & 1.37982 \\ \mathrm{H} & 1.40445 & -1.05070 & -0.90541 \\ \mathrm{C} & 0.29168 & 0.61829 & -0.26117 \\ \mathrm{C} & -0.96056 & 1.18506 & -0.57823 \\ \mathrm{C} & -1.17891 & 2.55588 & -0.36110 \\ \mathrm{C} & -0.14580 & 3.37110 & 0.13528 \\ \mathrm{C} & 1.11009 & 2.80737 & 0.42378 \\ \mathrm{C} & 1.32751 & 1.43206 & 0.24002 \\ \mathrm{H} & -1.74256 & 0.55313 & -1.00642 \\ \mathrm{H} & -2.15071 & 2.99198 & -0.60586 \\ \mathrm{H} & -0.31624 & 4.43913 & 0.29026 \\ \mathrm{H} & 1.91834 & 3.43558 & 0.80664 \\ \mathrm{H} & 2.29331 & 0.98147 & 0.48502\end{array}$

6 cis 1 conformer MP2/aug-cc-pVDZ T.E. $=-777.698838713$ a.u.

$\begin{array}{lccc}\mathrm{S} & -0.95066 & -1.99849 & 1.06080 \\ \mathrm{~N} & 1.22145 & -3.00765 & -0.12978 \\ \mathrm{~N} & 0.97332 & -0.74433 & -0.37224 \\ \mathrm{C} & 0.43624 & -1.90165 & 0.14890 \\ \mathrm{H} & 1.72924 & -3.01331 & -1.00941 \\ \mathrm{H} & 0.78636 & -3.88839 & 0.11903 \\ \mathrm{H} & 1.96553 & -0.78365 & -0.58953 \\ \mathrm{C} & 0.44410 & 0.56026 & -0.18919 \\ \mathrm{C} & -0.89150 & 0.84924 & -0.52490 \\ \mathrm{C} & -1.37122 & 2.15899 & -0.37066 \\ \mathrm{C} & -0.52579 & 3.18298 & 0.09495 \\ \mathrm{C} & 0.81155 & 2.89023 & 0.41272 \\ \mathrm{C} & 1.29509 & 1.57774 & 0.28068 \\ \mathrm{H} & -1.53856 & 0.05380 & -0.89580 \\ \mathrm{H} & -2.41030 & 2.38222 & -0.62568 \\ \mathrm{H} & -0.90626 & 4.20113 & 0.20721 \\ \mathrm{H} & 1.47680 & 3.67769 & 0.77584 \\ \mathrm{H} & 2.32885 & 1.33900 & 0.54880\end{array}$

6 cis 2 conformer MP2/aug-cc-pVDZ T.E. $=-777.697961677$ a.u.

$\begin{array}{lrrr}\mathrm{S} & -1.22409 & 2.05916 & 0.54862 \\ \mathrm{~N} & 1.09138 & 3.04733 & -0.32795 \\ \mathrm{~N} & 0.95505 & 0.75624 & -0.38020 \\ \mathrm{C} & 0.32320 & 1.93104 & -0.05817\end{array}$




$\begin{array}{rrrr}\mathrm{H} & 2.10300 & 2.96531 & -0.33705 \\ \mathrm{H} & 0.72275 & 3.90713 & 0.06007 \\ \mathrm{H} & 1.93084 & 0.81360 & -0.65536 \\ \mathrm{C} & 0.44293 & -0.55629 & -0.19900 \\ \mathrm{C} & 1.26620 & -1.51543 & 0.42057 \\ \mathrm{C} & 0.81683 & -2.83882 & 0.56094 \\ \mathrm{C} & -0.46210 & -3.19961 & 0.10254 \\ \mathrm{C} & -1.27970 & -2.23393 & -0.51232 \\ \mathrm{C} & -0.83146 & -0.91368 & -0.67700 \\ \mathrm{H} & 2.25041 & -1.22067 & 0.79696 \\ \mathrm{H} & 1.46001 & -3.58096 & 1.04060 \\ \mathrm{H} & -0.81818 & -4.22587 & 0.22119 \\ \mathrm{H} & -2.27176 & -2.51124 & -0.87772 \\ \mathrm{H} & -1.45486 & -0.16397 & -1.16385\end{array}$

6 TS1 conformer MP2/aug-cc-pVDZ T.E. $=-777.687394440$ a.u $(-93.5 \mathrm{~cm}-1)$

$\begin{array}{lrrr}\mathrm{S} & -0.83580 & 2.17784 & -1.02985 \\ \mathrm{~N} & 0.54762 & 2.66381 & 1.19924 \\ \mathrm{~N} & 1.18808 & 0.72679 & 0.14031 \\ \mathrm{C} & 0.30390 & 1.87462 & 0.12728 \\ \mathrm{H} & 1.25980 & 2.38531 & 1.86320 \\ \mathrm{H} & 0.03384 & 3.52850 & 1.31383 \\ \mathrm{H} & 1.84895 & 0.82278 & -0.63242 \\ \mathrm{C} & 0.54269 & -0.54166 & 0.07441 \\ \mathrm{C} & -0.61124 & -0.78694 & 0.84461 \\ \mathrm{C} & -1.19863 & -2.06107 & 0.83746 \\ \mathrm{C} & -0.64089 & -3.10242 & 0.07307 \\ \mathrm{C} & 0.51058 & -2.85428 & -0.69376 \\ \mathrm{C} & 1.09717 & -1.57784 & -0.69953 \\ \mathrm{H} & -1.04838 & 0.01845 & 1.44112 \\ \mathrm{H} & -2.09680 & -2.24070 & 1.43410 \\ \mathrm{H} & -1.10103 & -4.09328 & 0.07182 \\ \mathrm{H} & 0.95036 & -3.65096 & -1.29942 \\ \mathrm{H} & 1.98876 & -1.38513 & -1.30463\end{array}$

6 TS2 conformer MP2/aug-cc-pVDZ T.E. $=-777.681739577$ a.u $(-83.8 \mathrm{~cm}-1)$

$\begin{array}{lrrr}\mathrm{S} & -0.57006 & 2.93893 & 0.56855 \\ \mathrm{~N} & 0.69439 & 1.76333 & -1.45922 \\ \mathrm{~N} & 0.91779 & 0.67588 & 0.60182 \\ \mathrm{C} & 0.36132 & 1.77817 & -0.13712 \\ \mathrm{H} & 1.08138 & 0.91887 & -1.86592 \\ \mathrm{H} & 0.27804 & 2.45465 & -2.07209 \\ \mathrm{H} & 1.93744 & 0.68915 & 0.58378 \\ \mathrm{C} & 0.38724 & -0.60660 & 0.31146 \\ \mathrm{C} & -0.96437 & -0.75499 & -0.06674 \\ \mathrm{C} & -1.48892 & -2.03708 & -0.29658 \\ \mathrm{C} & -0.68468 & -3.18123 & -0.14953 \\ \mathrm{C} & 0.66087 & -3.03032 & 0.23065 \\ \mathrm{C} & 1.19730 & -1.75272 & 0.45586 \\ \mathrm{H} & -1.59975 & 0.12965 & -0.16113 \\ \mathrm{H} & -2.53815 & -2.13904 & -0.58623 \\ \mathrm{H} & -1.09990 & -4.17582 & -0.32728 \\ \mathrm{H} & 1.30060 & -3.90884 & 0.34949 \\ \mathrm{H} & 2.24405 & -1.64134 & 0.75694\end{array}$

